\title{
DERECHOS DEL PACIENTE Y EUTANASIA EN CHILE
}

\author{
Alejandra Zúñiga Fajuri*
}

"Las personas tienen el derecho fundamental a que se las deje en paz (...) ser libre significa tener derecho a elegir equivocada y hasta trágicamente" (Engelhardt, H. Tristram. Los fundamentos de la bioética).

\section{RESUMEN}

En este trabajo se analizan, en primer lugar, algunos de los dilemas morales asociados a los nuevos modos de morir que han surgido con el desarrollo de la técnica médica en los últimos años. Sin duda que la posibilidad de postergar artificialmente la muerte plantea cuestiones éticas relevantes tanto para quienes desean seguir viviendo como para quienes prefieren, en cambio, morir libres de las "interferencias" de la medicina moderna. En segundo lugar, este ensayo revisa los distintos conceptos de eutanasia y los problemas que la actual redacción del Proyecto de Ley de Derechos del Paciente - que "Regula los Derechos y Deberes que tienen las personas en relación con acciones vinculadas a su atención de Salud"-pudiera generar al momento de su aplicación en determinados casos límite.

\section{EUTANASIA - DERECHOS DEL PACIENTE - BIOÉTICA}

\section{Patient's rights and euthanasia in Chile}

\begin{abstract}
This essay review, first of all, some of the moral dilemmas usually associated to the new possibilities of dying that have arisen through the development of the medical technique in the last few years. There are important ethical issues related with the possibility of delaying artificially the death. That is true for those who wish to continue living but also for those who choose to die without the "interferences" of the medical advances. Secondly, this paper discusses some different concepts of euthanasia and the problems associated to the law project of Patient's Rights, especially in some difficult cases.
\end{abstract}

\section{EUTHANASIA - PATIENT'S RIGHTS - BIOETHICS}

* Abogada, Doctora en Derecho, Profesora e investigadora de la Escuela de Derecho de la Universidad de Valparaíso, Errázuriz 2120. Valparaíso, Chile, alejandra.zuniga@uv.cl. Artículo recibido el 8 de junio de 2008 y aceptado para su publicación por el Comité Editorial el 24 de octubre de 2008. 


\section{INTRODUCCIÓN}

$\mathrm{E}$ 114 de marzo de 2007, la española Inmaculada Echeverría logró al fin lo que "tanto anhelaba: Morir". Inmaculada dejó, a los 51 años, una vida que dependía de un respirador mecánico del que luchó por separarse por más de 20 años y del que se ha desprendido, no sin incitar el debate legal, ético y médico en torno a la eutanasia. Lo mismo ocurrió en el caso de Piergiorgio Welby, un enfermo con distrofia muscular progresiva, quien murió en diciembre del año 2006 cuando su médico Mario Riccio, a petición suya y tras sedarle, le desconectó del respirador. Pero ¿fue una eutanasia lo que se practicó a Echeverría y a Piergiorgio Welby o fue tan sólo la consecuencia del libre ejercicio de un derecho que se conoce desde hace mucho: el derecho a rechazar o suspender un tratamiento médico? ¿Es el rechazo o suspensión de un tratamiento un tipo de eutanasia?

En este trabajo se analizan, en primer lugar, algunos de los dilemas morales asociados a los nuevos modos de morir que han surgido con el desarrollo de la técnica médica en los últimos años. Sin duda que la posibilidad de postergar artificialmente la muerte plantea cuestiones éticas relevantes tanto para quienes desean seguir viviendo como para quienes prefieren, en cambio, morir libres de las "interferencias" de la medicina moderna. En segundo lugar, este ensayo revisa los distintos conceptos de eutanasia y los problemas que la actual redacción del Proyecto de Ley que "Regula los derechos y deberes que tienen las personas en relación con acciones vinculadas a su atención de salud" -también conocido como Proyecto de Ley de Derechos del Paciente- pudiera generar al momento de su aplicación en determinados casos límite.

\section{LOS PROBLEMAS DEL NUEVO MODO DE MORIR. Derechos DEL PACIENTE Y EUTANASia}

A pesar de lo intensa que puede llegar a ser la angustia emanada de la idea de la propia muerte ésta, sin embargo, resulta a veces desplazada por los padecimientos y tormentos que puede significar sostener la propia vida. Es el miedo al dolor lo que nos hace odiar la idea de la muerte y también el miedo al dolor lo que nos hace, en ciertas circunstancias, desearla. Todo es, finalmente, una cuestión de intensidad de sufrimiento. Inmaculada Echeverría Ramírez, quien padecía de una severa distrofia muscular progresiva, confesó que desde los 29 años tuvo claro que quería morir. ${ }^{1}$ El gran debate moral que se produjo en España a propósito de su caso fue en gran medida producto de la decisión del Consejo Consultivo de la Comunidad Autónoma de Andalucía de autorizarla a "renunciar al tratamiento médico" que la mantenía con vida, ${ }^{2}$ reconociéndose

${ }^{1}$ Diario El País, 18/10/2006.

${ }^{2}$ Echeverría solicitó al Consejo Consultivo de Andalucía que permitiera que se la desconectase del ventilador mecánico que la mantenía con vida. Tras una resolución favorable, emitida por la Comisión Autonómica de Ética e Investigación Sanitaria, el Consejo Consultivo de Andalucía, en su dictamen número 
así el derecho de los pacientes, no sólo a consentir un tratamiento, sino que también a suspenderlo.

Si bien para algunos el caso Echeverría sería "un tipo de eutanasia" que no debiera estar autorizada por la ley, la gran mayoría de médicos y juristas coinciden en que rechazar la respiración asistida no es un supuesto de eutanasia, sino que un derecho que tienen todas las personas en el marco de la legislación española, en particular la Ley de Autonomía del Paciente de 2002, que señala que "todo paciente tiene derecho a negarse al tratamiento". Así, muchos de los especialistas que conocieron del caso destacaron la distinción que existe entre la petición de, por ejemplo, que se inyecte a alguien un fármaco que le quite la vida - lo que sí sería considerado eutanasia- y la renuncia a un tratamiento médico como la respiración asistida. Limitar el esfuerzo terapéutico, sostenían, sería legal pues se diferencia claramente de una eutanasia, que implicaría una "práctica activa para acabar con la vida de alguien", lo que "no ocurre cuando una persona señala su deseo de renunciar a un tratamiento que, como consecuencia, acelera su muerte"3 ¿Qué habría ocurrido si la paciente hubiera denegado desde el principio conectarse a un respirador? El debate surge, luego, en torno a si ha sido un tipo de eutanasia o simplemente la negativa lícita a seguir recibiendo un tratamiento "gravoso". ${ }^{4}$ Con todo, hubo quienes calificaron el caso de Inmaculada Echeverría como "eutanasia pura y dura” pues la legislación actual sólo permitiría retirar el "esfuerzo terapéutico" cuando "un paciente no tiene salida" y sólo representa un "ensañamiento inútil sobre su salud". 5

De igual forma, el debate sobre Piergiorgio Welby surgió cuando la jueza del Tribunal de Roma Zaira Secchi determinó, en octubre de 2007, que el médico no cometió delito alguno al desconectar al paciente del respirador pues rechazar una terapia sanitaria no deseada es un "derecho reconocido en la Constitución" italiana. La sentencia expuso que la actuación del anestesista Riccio "no fue homicidio pues (...) el médico actuó ateniéndose al derecho de la víctima a privarse de un tratamiento no deseado, un derecho reconocido por la Constitución respecto al cual el médico asumió el deber jurídico de consentir". Para la jueza hablar en este caso de eutanasia sería "tergiversador". 6

90/07, casi por unanimidad, determinó que ambos comportamientos eran conformes a derecho. El Consejo estimó amparadas jurídicamente ambas conductas por la Jurisprudencia (diversas Sentencias del Tribunal Constitucional), por el Derecho supranacional (Declaración Universal de Derechos Humanos y Convenio de Oviedo), por el Derecho comparado (Ley Fundamental de la República Federal de Alemania y Constituciones italiana y portuguesa) y por determinadas leyes estatales (la Constitución Española, la Ley 41/2002 y el Código Penal) y autonómicas (Ley 2/1998, de Salud de Andalucía).

${ }^{3}$ Opinión del presidente del Comité de Deontología de la Organización Médica Colegial, Rogelio Altisent, y de la médica y bioética Margarita Iraburu. Diario El País, 19/10/2006.

${ }^{4}$ Opinión de José Miguel Serrano, profesor de Filosofía del Derecho de la Universidad Complutense de Madrid. Diario El Mundo, 15/03/2007.

${ }^{5}$ Opinión de Natalia López Moratalla, doctora en Ciencias Biológicas y catedrática de Bioquímica de la Universidad de Navarra. Diario El Mundo, 15/03/2007.

6 "Jueza exculpa a un médico del delito de eutanasia", en: www.deia.com/es/impresa/2007/10/20/ bizkaia/gizartea/410050.php. 
La intervención creciente de la técnica médica ha significado un cambio importante en el modo tradicional de morir. La sociedad moderna se ve cada día enfrentada a más y mayores retos relacionados con la posibilidad que tiene hoy un gran número de personas de decidir, en mayor o menor medida, el momento y el modo en que habrá de producirse su propia muerte. Esta realidad, que ha introducido procedimientos "tecnologizados" de morir, nos obliga a repensar el tema de la disponibilidad de la propia vida y de qué significa la "buena muerte" o eutanasia. El progreso de las técnicas médicas ha hecho que la muerte hoy sufra una especial transformación. El filósofo Víctor Méndez ${ }^{7}$ ha concebido esta realidad contemporánea como un cambio radical en el modo de entender la muerte que la ha convertido "desde el hecho inevitable que era antes en una especie de enfermedad contra la que hay que luchar y a la que se puede vencer (...) El lugar preeminente que tenía el destino en el modo tradicional de morir lo ocupa aquí la técnica. Y el lugar que ocupaba antes la aceptación del orden lo llenan ahora las esperanzas depositadas en la misma. (...) La muerte, en el contexto hospitalario, y desde el punto de vista de la ciencia, parece llegar más como el resultado de un fracaso técnico que como un acontecimiento que tiene un lugar determinado e inamovible dentro de un orden de la naturaleza". 8 Así, la implantación del modo tecnológico de morir ha implicado también un aumento drástico del número de decisiones sociales que han de ser tomadas durante ese proceso.

Esta realidad, que Méndez denomina la "racionalización de la muerte", supone entrar en la discusión asociada a la aparición de los diferentes sistemas médicos de mantenimiento artificial de las funciones del cuerpo humano. Dentro de ese conjunto de posibilidades técnicas de sustitución de las funciones del cuerpo -que condujo a las modernas unidades de cuidados intensivos- cabe incluir, por ejemplo, aparatos como el respirador artificial, el cual sustituye la función pulmonar; las máquinas de hemodiálisis, que sustituyen las funciones del riñón; las técnicas de hidratación y de alimentación artificial, que permiten reemplazar el funcionamiento del sistema digestivo; las técnicas de reanimación cardiaca mediante estimulación externa que se conocen muy impropiamente con el nombre de "resucitación”, las cuales permiten recuperar el funcionamiento del corazón; y también las técnicas de trasplante de órganos que permiten sustituir un órgano propio dañado por otro ajeno, entre otros ejemplos. De esta manera se fue imponiendo cada vez con mayor fuerza la idea de que el proceso de morir de un individuo en nuestra sociedad depende, en gran medida, de la decisión médica de aplicar o no una de esas técnicas.

Existe indudablemente una línea de crecimiento de la tolerancia estatal hacia ciertas conductas relacionadas con el proceso de morir que se ha venido manifestando en casi todo el mundo occidental durante el último tiempo. La tolerancia social hacia las conductas que ponen fin a la vida de enfermos graves por petición de los mismos, aunque bastante generalizada, ha dado un paso más allá y se ha convertido en autorización legal

\footnotetext{
${ }^{7}$ Méndez, V., Eutanasias, derechos, razones, Trotta, Madrid, 2001. Capítulos II y III.

${ }^{8}$ Ibid., pp. 28-29.
} 
explícita de esas prácticas en países como Holanda, Bélgica, Australia y el estado de Oregon en Estados Unidos. ${ }^{9}$ El derecho a elegir el modo de morir tendría su origen en lo que Dworkin llama "el rasgo más relevante de la cultura política occidental”, implica la creencia en la dignidad humana individual, es decir, en la idea de que "las personas tiene el derecho y la responsabilidad moral de enfrentarse por sí mismas a las cuestiones fundamentales acerca del significado y valor de sus propias vidas, respondiendo a sus propias conciencias y convicciones". ${ }^{10}$

Ahora, ¿son todos los casos mencionados - de intervención médica en el proceso de morir-iguales? ¿Qué es, al fin, la eutanasia? El concepto evidentemente no es pacífico, aunque se está de acuerdo en general en definirla etimológicamente como "buena muerte" (del griego eu, bien, bueno, y thanatos, muerte). Para Romeo Casabona, la eutanasia es "la privación de la vida de otra persona realizada por razones humanitarias, a requerimiento del interesado, que sufre una enfermedad terminal incurable o una situación de invalidez irreversible en el estado actual de la ciencia médica y desea poner fin a sus sufrimientos, así como las situaciones en que aquél no puede manifestar su voluntad o no puede ser tenida en cuenta por cualquier motivo". ${ }^{11}$ La eutanasia admite diversas clasificaciones. Según Romeo, es posible distinguir, atendiendo a la ejecución, entre eutanasia activa o pasiva. La primera consiste en realizar actos para ayudar a morir eliminando o aliviando el sufrimiento, mientras que la segunda supone dejar de hacer aquello que permitiría

${ }^{9}$ La primera legislación en el mundo que estableció explícitamente un derecho del paciente a determinar el momento de su muerte fue en el territorio norte de Australia donde se promulgó, en 1996, una Ley de derechos de los enfermos terminales que permitió a los pacientes con una esperanza de vida menor de seis meses el auxilio médico para morir. Con todo, esta ley estuvo vigente tan sólo nueve meses pues fue derogada en 1997 por el Gobierno Federal. El mismo año que en Australia, el estado de Oregón, en Estados Unidos, promulgó su Ley de muerte con dignidad que permite a todo enfermo terminal, con una esperanza de vida también menor de seis meses, mayor de edad y residente en el estado, solicitar que se le proporcione la medicación necesaria para acabar con su vida. Esta ley, que aún está vigente, inició en el país un gran debate moral que continúa hasta hoy. Por su parte, la Corte Constitucional de Colombia dictó en mayo de 1997 una sentencia en la que invocando el principio constitucional de la solidaridad y el derecho fundamental al desarrollo de la libre personalidad, consideró inconstitucional la punición del acto médico de provocar la muerte de un enfermo terminal que da su consentimiento para ello. Con todo, a pesar de que la Corte exhorta al legislativo colombiano para que resuelva mediante una ley el tema de la "muerte digna" ello aún no ha ocurrido. Recientemente, en Holanda, se reformó la legislación con la polémica Ley de terminación de la vida a petición propia y del auxilio al suicidio que, modificando el Código Penal, dispone la despenalización de las conductas de acabar con la vida de otro a su requerimiento y del auxilio al suicidio cuando se realice por un médico y según las indicaciones que marca la ley. En Holanda, con todo, ya desde la década del setenta se venían realizando estas prácticas autorizadas por la jurisprudencia, lo cual en 1984 culminó con la decisión del Tribunal Supremo de consolidar una doctrina que apreciaba la eximente del estado de necesidad en el caso del médico que causaba la muerte de un paciente que lo solicitaba a consecuencia de sufrimientos graves e insoportables sumados a la imposibilidad de recuperación. Por último, la ley belga de 22 de junio de 2002, siguiendo el ejemplo holandés, ha modificado la ley penal creando una legislación que permite, bajo estrictos presupuestos, la eutanasia y el auxilio médico al suicidio. Méndez, V., ob. cit., p. 47.

${ }^{10}$ Dworkin, R., El Dominio de la Vida, Una discusión acerca del aborto, la eutanasia y la libertad individual, Ariel, Barcelona, 1994, p. 217.

${ }^{11}$ Romeo, C. M., El derecho y la bioética ante los límites de la vida humana, Editorial Centro de Estudios Ramón Areces, Madrid, 1994, pp. 424-5. 
alargar la vida. A su vez, atendiendo a la intencionalidad del autor, puede ser directa o indirecta. La eutanasia directa persigue el acortamiento de la vida del paciente mediante actos positivos (ayuda a morir); la indirecta tiene un doble efecto: aliviar el sufrimiento del paciente abreviando al mismo tiempo su vida, que es el efecto secundario derivado de aquel objetivo principal (por ejemplo, la administración de morfina tiene efectos perjudiciales por depresión de la función respiratoria que en altas dosis puede acelerar la muerte). ${ }^{12}$

Es la eutanasia pasiva la que, probablemente, presenta más problemas éticos y jurídicos pues no resulta fácil establecer los límites entre lo lícito y lo ilícito y, por lo general, existe una confusión sobre lo que realmente es una omisión eutanásica y lo que únicamente significa la interrupción del tratamiento en determinadas situaciones. Asimismo, las dificultades para diferenciar entre eutanasia pasiva indirecta y la suspensión de tratamientos vitales emanan de una construcción argumentativa, para muchos sospechosa, que intenta distinguir actos y omisiones que en esencia parecen ser la misma cosa. Así, para R.G. Frey, este tipo de clasificaciones son artificiales. "Desde un punto de vista causal podemos ver en esta reflexión la línea divisoria que toda la bibliografía traza entre el auxilio médico al suicidio y la eutanasia voluntaria activa. Esta línea depende de quien actúa en último lugar en la cadena que provoca la muerte: si el último en actuar es el paciente que toma la pastilla que le ha dado el médico, entonces tenemos un caso de auxilio médico al suicidio, mientras que si el médico es quien actúa en último lugar inyectando la morfina, entonces se trata de un caso de eutanasia voluntaria activa". ${ }^{13}$

Con todo, si bien para la mayoría de los autores parece claro que no es lo mismo dar una pastilla a un enfermo para permitirle morir que desconectarle del respirador mecánico -lo que hace que los casos de Echeverría y Piergiorgio Welby sean distintos del conocido caso de Diane Pretty-, ${ }^{14}$ Peter Singer considera necesaria una clasificación alternativa que distingue tan sólo entre eutanasia voluntaria, involuntaria y no voluntaria. La primera, la eutanasia voluntaria, es aquella que se lleva a cabo a petición de la persona

12 Ibid., p. 421.

${ }^{13}$ Frey, R. G., "Distintos tipos de muerte", en La Eutanasia y el Auxilio Médico al Suicidio, Dworkin, G./Frey, R. G./Bok, S. Cambridge University Press, Madrid, 2000. p. 50.

${ }^{14}$ Diane Pretty recurrió a los tribunales ingleses a los 43 años de edad para que le permitieran ser auxiliada por su marido al suicidio. Es decir, para que se la autorizara a la eutanasia activa, salvando a su marido de la cárcel. El Tribunal Europeo de Derechos Humanos, ante el cual recurrió Pretty la decisión de estos tribunales, consideró que la normativa inglesa sobre asistencia al suicidio era compatible con las normas de la Convención Europea de Derechos Humanos pues los Estados tendrían derecho a seguir los pasos que estimaran pertinentes para salvaguardar el derecho a la vida de sus nacionales. Sobre la supuesta violación del art. $2^{\circ}$ del Convenio, que protege el derecho a la vida, el tribunal dispuso que no era posible interpretar dicha norma en el sentido que ella estableciera, como alegaban los demandantes, un aspecto negativo que también fuera digno de protección: el derecho a elegir el momento y el modo de la propia muerte. La libertad, entonces, protegida por la Convención no incluiría el derecho a la muerte. Sobre la violación del art. $3^{\circ}$, que prohíbe a los Estados someter a sus ciudadanos a torturas u otros tratos crueles, inhumanos y degradantes, el Tribunal Europeo consideró que Diane Pretty estaba sufriendo a consecuencia del desarrollo natural de su enfermedad y no de una acción de la que pudiese hacerse responsable al Estado (Case of Pretty $v$. The United Kingdom, Tribunal Europeo de Derechos Humanos, Strasbourg, 29-04-2002). 
que va a morir. "Algunas veces la eutanasia voluntaria apenas puede distinguirse del suicidio asistido". ${ }^{15}$ La eutanasia involuntaria se produce cuando la persona que muere tiene capacidad para consentir a su propia muerte, pero no lo hace, bien porque no le preguntan, bien debido a que cuando se le pregunta la persona decide seguir viviendo. "Matar a alguien que no ha dado su consentimiento para morir solamente podrá considerarse eutanasia cuando el motivo para matar es el deseo de que acabe un sufrimiento insoportable para la persona que va a morir". Con todo, Singer considera que no existe justificación posible para este tipo de eutanasia. ${ }^{16}$ Por último, la eutanasia será no voluntaria cuando un ser humano no es capaz de entender la elección entre la vida y la muerte. "Entre los que no pueden dar su consentimiento se incluirían los enfermos incurables o los recién nacidos con graves discapacidades, y las personas que debido a accidentes, enfermedades o avanzada edad han perdido permanentemente la capacidad para entender el tema en cuestión, sin haber pedido o rechazado previamente la eutanasia en estas circunstancias". ${ }^{17}$ Ahora, ¿qué tipo de legislación se quiere establecer en Chile en esta materia?

\section{Los proyectos de Ley de Derechos del Paciente}

La Organización Mundial de la Salud distingue tres modos de incorporar a las legislaciones nacionales los derechos de los pacientes. Algunos países han promulgado una ley única integral sobre derechos del paciente, como Finlandia, Uruguay, Países Bajos, Israel, Argentina, Islandia, Dinamarca y Noruega -todos en la década del 90-. Otros han integrado los derechos de los pacientes en la legislación que reglamenta el sistema de atención de salud o en varias leyes sanitarias, como Canadá, Grecia, Austria o Estados Unidos. Finalmente, las declaraciones o cartas sobre los derechos de los pacientes que tienen una condición jurídica variable como política nacional y que a menudo se incorporan en los reglamentos por los que se rigen los establecimientos de atención sanitaria, se han considerado más adecuados para las tradiciones jurídicas de países como Francia, Irlanda, Reino Unido y Portugal. Los derechos clásicos de los pacientes son el consentimiento informado, el acceso a los registros médicos y el carácter confidencial de los datos. ${ }^{18}$

En Chile el reconocimiento de los derechos de los pacientes se ha intentado en el contexto de la Reforma a la Salud AUGE. Con todo, la polémica en torno al proyecto de ley "Sobre los derechos y deberes de las personas en salud" del año 2001 obligó a que la autoridad decidiera finalmente su archivo. La consagración legal del "consentimiento informado" y la regulación de la muerte digna, centrada en la posibilidad de evitar el

\footnotetext{
${ }^{15}$ Singer, P., Ética práctica, Cambridge University Press, Gran Bretaña, 1995. Pp. 217 y sig.

${ }^{16}$ Ibid., pp. 248 y sig.

${ }^{17}$ Ibíd., pp. 248 y sig.

18 Organización Mundial de la Salud, Informe sobre la Salud en el Mundo 2000, Mejorar el desempeño de
} los sistemas de salud, p. 151. 
ensañamiento u obstinación terapéutica, atrajo el interés de académicos, profesionales y religiosos que, en algunos casos, vieron aquí la puerta de entrada a la instauración de la eutanasia. El proyecto original al referirse al derecho al consentimiento informado disponía: "Toda persona tiene derecho a otorgar o denegar su consentimiento para someterse a cualquier procedimiento invasivo, de cirugía mayor o compleja, y otros que defina el Ministerio de Salud, salvo que la negativa pueda implicar su muerte y siempre que no exista otro procedimiento alternativo". ${ }^{19}$

A continuación se establecía que "No obstante lo dispuesto en el inciso primero del artículo 16, si la voluntad del paciente de rechazar los procedimientos y cirugías indicados en el referido inciso puede implicar su muerte, dicha voluntad será acatada siempre que se cumplan los siguientes requisitos: a) Que se trate de un paciente en estado terminal, esto es, cuando padezca un precario estado de salud, producto de una lesión corporal o una enfermedad grave e incurable, que haga prever que le queda muy poca expectativa de vida; b) Que los cuidados que se le puedan brindar al paciente sean innecesarios, en cuanto éstos sólo persigan prolongar su agonía; y c) Que el médico tratante consulte la opinión de un segundo médico, que no haya participado en la atención del paciente, el cual deberá estar conteste con él, tanto en la calidad de terminal del estado de salud, así como en la innecesariedad de los cuidados". ${ }^{20}$

El proyecto proponía al médico que, al acatar la decisión del paciente de suspender su tratamiento médico, simplemente "dejara que el curso de la enfermedad siguiera su evolución natural” hasta que se produzca la muerte. ¿Se trataba -como denunciaron algunos- de un caso de eutanasia? En el mensaje del proyecto - previendo, probablemente, los conflictos morales que más de alguno vería en esta nueva institución- sus redactores argumentaban que "el médico no tiene la intención de matar al paciente cuando, a petición de este último, suprime los cuidados no debidos, no necesarios y que no tienen sentido. Esta regulación no debe confundirse con la eutanasia". ${ }^{21}$ ¿Por qué no debiéramos confundirla? La eutanasia, en el entender de los legisladores, "implica poner fin voluntariamente a la vida de una persona enferma, con miras a evitarle intensos sufrimientos". ${ }^{22}$

Según revisamos con Romeo Casabona, esta definición de eutanasia incluiría el caso de la eutanasia activa (que supone una acción) directa (para provocar la muerte) y la eutanasia activa indirecta (que 'pretende sólo' evitar un sufrimiento, aunque sea mediante una acción que acarreará seguramente la muerte del paciente). ¿Y la eutanasia pasiva? Ella comprende distintas situaciones dentro de las cuales se encuentra -según veremos- aquella derivada del derecho al rechazo o suspensión de los tratamientos vitales. Visto desde el punto de vista del médico tratante, éste omite (eutanasia pasiva) aquellos tratamientos que prolongarían la vida del paciente. Para Pablo de Lora, cuando

${ }^{19}$ Artículo 16. Proyecto de ley Sobre los derechos y deberes de las personas en materia de salud del año 2001. Boletín 2727-11.

${ }^{20}$ Ibid., art. 18.

${ }^{21}$ Ibid.

${ }^{22}$ Ibid. 
el móvil de la acción consiste en paliar el sufrimiento padecido, sabiendo que con gran probabilidad, cuando no con total seguridad, ello hará que se acelere la muerte, nos encontramos ante la modalidad llamada eutanasia indirecta. "Por definición la eutanasia indirecta es siempre activa pues no cabe, no haciendo nada, disminuir el dolor a quien lo sufre (cabe, eso sí, no añadir sufrimiento mediante métodos encarnizadores de prolongación de una vida irremediablemente terminal)". ${ }^{23}$ Luego, no se ocultaría el hecho de que quien "suspende" un tratamiento vital, sin intención de producir la muerte, está actuando y no omitiendo.

El nuevo proyecto alternativo presentado en agosto del 2006, que "Regula los derechos y deberes que tienen las personas en relación con acciones vinculadas a su atención de Salud", buscó explicitar el contenido de las mismas instituciones mencionadas en el primer proyecto, esto es, el derecho al Consentimiento Informado y el llamado Testamento Vital. ${ }^{24}$ Así, el proyecto comienza resaltando su objetivo de velar por la dignidad de las personas, lo que se traduce en el respeto por su autonomía y control sobre el propio cuerpo. Se buscaría reconocer un espacio de soberanía personal, sin ir más allá de los consensos nacionales e internacionales sobre la materia. En ese contexto reconoce, en los artículos 14 y siguientes, el "Derecho de las personas a decidir informadamente", lo cual no es más que la consagración del derecho de toda persona a otorgar o denegar su voluntad para someterse a cualquier procedimiento o tratamiento vinculado a su atención de salud. "El ejercicio de este derecho es libre, voluntario, expreso e informado". ${ }^{25}$

Cabe destacar que el "derecho a la decisión informada" es de antigua data y que ha sido reconocido en la Declaración de Lisboa de la Asociación Médica Mundial (AMM) sobre los Derechos del Paciente donde, junto con enumerar los derechos principales del paciente que la profesión médica ratifica y promueve, dispone: "Los médicos y otras personas u organismos que proporcionan atención médica, tienen la responsabilidad conjunta de reconocer y respetar estos derechos. Cuando la legislación, una medida del gobierno, o cualquier otra administración o institución niega estos derechos al paciente, los médicos deben buscar los medios apropiados para asegurarlos o restablecerlos". ${ }^{26}$ Otro de los principios destacados en la Declaración es el relacionado con el derecho a la

23 De Lora, P., Entre el vivir y el morir. Ensayos de Bioética y Derecho, Fontamara, México, 2003, p. 82.

24 Tras ingresar al Congreso en agosto de 2006, el proyecto fue votado por la Cámara de Diputados, el 31 de julio de 2007. Aunque fue aprobado en general, no se logró el quórum necesario para artículos como el de la confidencialidad de la ficha clínica, por lo que al presentarse múltiples indicaciones regresó a la comisión de Salud que debe preparar un nuevo informe. El proyecto tiene urgencia simple, por lo que no hay aún una fecha estimada para que se convierta en ley, en consideración a que todavía debe ser estudiado por el Senado, una vez aprobado el texto definitivo por la Cámara de Diputados.

${ }^{25}$ Proyecto de ley que "Regula los derechos y deberes que tienen las personas en relación con acciones vinculadas a su atención de Salud". Boletín No 4398-11.

${ }^{26}$ Asociación Médica Mundial (AMM), Declaración de Lisboa sobre los Derechos del Paciente. Adoptada por la $34^{\mathrm{a}}$ Asamblea Médica Mundial Lisboa, Portugal, septiembre/octubre 1981, enmendada por la $47^{\mathrm{a}}$ Asamblea General Bali, Indonesia, septiembre 1995, y revisada su redacción en la 171 a Sesión del Consejo, Santiago, Chile, octubre 2005. 
autodeterminación que sostiene que: "El paciente tiene derecho a la autodeterminación y a tomar decisiones libremente en relación a su persona. El médico informará al paciente las consecuencias de su decisión. El paciente adulto mentalmente competente tiene derecho a dar o negar su consentimiento para cualquier examen, diagnóstico o terapia. El paciente tiene derecho a la información necesaria para tomar sus decisiones. El paciente debe entender claramente cuál es el propósito de todo examen o tratamiento y cuáles son las consecuencias de no dar su consentimiento". ${ }^{27}$

En la misma línea se menciona el derecho a la dignidad, estableciéndose que "la dignidad del paciente y el derecho a su vida privada deben ser respetadas en todo momento durante la atención médica y la enseñanza de la medicina, al igual que su cultura y sus valores. El paciente tiene derecho a aliviar su sufrimiento, según los conocimientos actuales. El paciente tiene derecho a una atención terminal humana y a recibir toda la ayuda disponible para que muera lo más digna y aliviadamente posible". ${ }^{28}$ Finalmente, junto con identificar los derechos fundamentales a la libertad y dignidad, se hace un llamado a evitar el "encarnizamiento terapéutico" sobre la base de realizar un análisis estricto de la proporcionalidad de los tratamientos médicos. De este modo, "Nadie está obligado a utilizar todas las intervenciones médicas actualmente disponibles, sino que aquellas que ofrecen una razonable probabilidad de beneficio en términos de preservar y/o recuperar la salud". ${ }^{29}$ Lo anterior significa que debe limitarse el esfuerzo terapéutico cuando, al menos, se dan algunas de las siguientes circunstancias: cuando los procedimientos atrasan la muerte, en vez de prolongar la vida; o cuando el sobretratamiento busca sólo mantener la vida biológica sin preocuparse por la calidad de la misma. Como norma general, entonces, la conducta terapéutica del médico debe ser proporcionada, vale decir, los beneficios esperados para un paciente con un determinado diagnóstico y pronóstico tienen que guardar relación con el esfuerzo terapéutico, los sufrimientos del paciente, los costos de toda índole involucrados y los riesgos asumidos. Por esta razón, la proporcionalidad en la acción médica es una exigencia no sólo técnica sino que también ética, pues no sería lícito llevar a cabo acciones diagnósticas o terapéuticas desmedidas. ${ }^{30}$

Por su parte, la Declaración para la promoción de los derechos de los pacientes en Europa, de 1994, promovida por la Organización Mundial de la Salud, dispone entre los "Principios de los Derechos de los Pacientes en Europa" aquel relacionado con el "consentimiento informado" de la siguiente manera: "3.1. El consentimiento informado del paciente es el requisito previo a toda intervención médica; 3.2. El paciente tiene el derecho a negarse o a detener una intervención médica. Las implicaciones de negarse a recibir o detener tal intervención deben ser cuidadosamente explicadas al paciente; 3.3. Cuando

\footnotetext{
27 Ibid.

${ }^{28}$ Ibid.

29 Tabeada, P., El principio de proporcionalidad terapéutica en las decisiones de limitar tratamientos. Boletín de la Escuela de Medicina de la Universidad Católica, No 28, 1998, pp. 17-23.

${ }^{30}$ Grupo de Estudio de Ética Clínica, Sobre las acciones médicas proporcionadas y el uso de métodos extraordinarios de tratamiento. Revista Médica de Chile, jun. 2003, vol. 131, N 6, pp. 689-696.
} 
el paciente sea incapaz de expresar su voluntad y se necesite urgentemente llevar a cabo una intervención, se puede presumir el consentimiento del paciente, a menos que resulte obvio por una declaración de voluntades anticipadas previa que en dicha situación el consentimiento sería denegado". 31

¿Cómo se desarrollan estos derechos en el proyecto de ley pendiente de discusión en el Congreso Nacional?

\section{i) Derecho al consentimiento informado: rechazar o suspender un tratamiento}

El proyecto dispone el principio de respeto de las personas en situación de salud terminal como una necesaria consecuencia de la consagración del derecho a consentir cualquier intervención que, en el ámbito de la propia salud, sea propuesta por los profesionales tratantes. Las personas en "estado terminal" pueden rechazar tratamientos que estimen desproporcionados e innecesarios, pues se pretende garantizar la no prolongación innecesaria de la agonía. Consagrar la "limitación del esfuerzo terapéutico", con el objeto de evitar el encarnizamiento terapéutico, parece constituir hoy un consenso bioético general, por lo que el proyecto busca resolver las dificultades teóricas y prácticas que supone distinguir con claridad las situaciones que, por una parte, van encaminadas a evitar actuaciones extraordinarias e innecesarias y, por otra, aquellas que desean acelerar artificialmente el proceso de muerte, ya sea mediante actos positivos cuyo objetivo es procurar la muerte o de la omisión deliberada de actuaciones razonables, necesarias u ordinarias. $^{32}$

Los "métodos extraordinarios" incluyen, en general, técnicas o instrumentos complejos, escasos, invasivos, de alto costo y cuyo uso expedito y sostenido requiere un entrenamiento especializado del equipo médico. Es el caso, por ejemplo, del ventilador mecánico, de los aparatos de hemodiálisis, del trasplante de órganos, etc. Cuando están indicados, porque se juzga razonablemente que su uso redundará en un claro beneficio vital para el paciente, se los considera procedimientos terapéuticos éticamente proporcionados y técnicamente útiles. En caso contrario, si se estima que de su aplicación no resultará ningún beneficio significativo para el enfermo, o incluso se piensa que sólo puede prolongar innecesariamente un esfuerzo terapéutico o un sufrimiento para el paciente, se convierten en procedimientos terapéuticos desproporcionados y fútiles. ${ }^{33}$

Los métodos extraordinarios de tratamiento plantean al médico dos dilemas: uno, el emplearlos o no emplearlos en un caso particular y, otro, tomar la decisión de suspenderlos una vez iniciada su aplicación. Así, la limitación o restricción del uso de métodos extraordinarios de tratamiento requiere determinar cuándo es legítimo y bueno para el

${ }^{31}$ Declaración para la promoción de los Derechos de los Pacientes en Europa. Consulta Europea sobre los Derechos de los Pacientes. Amsterdam, 28-30 de marzo de 1994. Oficina Regional para Europa, Organización Mundial de la Salud.

${ }^{32}$ Mensaje del proyecto de ley que "Regula los derechos y deberes que tienen las personas en relación con acciones vinculadas a su atención de Salud". Boletín N4398-11.

33 Grupo de Estudio de Ética Clínica. ob. cit., pp. 689-696. 
paciente abstenerse de algunas acciones terapéuticas. En la práctica clínica, para fundamentar la limitación de tratamientos se utilizan los siguientes criterios: a) Inutilidad del tratamiento: éticamente el médico no está obligado a administrar terapias inútiles o inefectivas; b) Deseos o voluntad del paciente: esto supone que el paciente sea competente, esté debidamente informado y comprenda la información que se le da. Si se cumplen estas condiciones, es válido el rechazo informado de un paciente a una determinada terapia; c) Calidad de vida: es el paciente quien -en última instancia- debe juzgar su calidad de vida, ya que es él quien está en mejores condiciones de examinar los diversos aspectos de su subjetividad, y d) Costo del procedimiento: se debe propender al uso racional y eficiente de los recursos médicos. ${ }^{34}$

El proyecto contempla, en su artículo 17, la situación de aquellas personas que fueren informadas, por el profesional tratante, de que su estado de salud es calificable como terminal, lo que se produciría cuando la persona padezca un precario estado de salud, producto de una lesión corporal o una enfermedad grave e incurable, y que los tratamientos que se le pueden ofrecer sólo tengan por efecto retardar innecesariamente la muerte. En este caso, la persona puede rechazar los tratamientos. Además se agrega que este rechazo, por una parte, no puede implicar como objetivo la aceleración artificial del proceso de muerte ni, tampoco, la renuncia al derecho a recibir los cuidados paliativos que permitan a la persona hacer más soportables los efectos de su enfermedad. ${ }^{35}$

¿Es este un caso de eutanasia? Para responder es necesario aclarar aún más algunos conceptos. Según la definición de Romeo, la eutanasia pasiva "consiste en la interrupción del tratamiento, en no iniciar otros o, en términos generales, en no prestar auxilio o asistencia, no evitando con todo ello el desenlace de la muerte, de modo que ésta no se hubiera producido o hubiera sido significativamente más adelante”. ${ }^{36}$ Este parece ser, en términos generales, el caso contemplado en el artículo 17, pues se permite a los enfermos terminales "rechazar" los tratamientos que impliquen retardar innecesariamente la muerte. Pero, ¿puede un enfermo terminal -según el proyecto en estudio- suspender o interrumpir un tratamiento? Aunque Romeo sigue estimando que se trataría de un tipo de eutanasia "pasiva", parece claro que en este caso ya no estaríamos ante una omisión del facultativo sino que frente a una acción por medio de la cual se pone fin a un tratamiento ya iniciado.

Si bien el articulado del proyecto de ley no hace sinónimos los términos "rechazo" "suspensión" e "interrupción”, sí lo hizo su mensaje, que dispone que "Es posible que una persona aquejada de una enfermedad incurable y en fase terminal, decida no seguir siendo sometida a tratamientos. En estas situaciones, ¿cómo puede ser humanamente procedente que una persona sea sometida a tratamientos indeseados, que no tendrán ningún resultado positivo o, al menos, con un grado importante de éxito? ¿Cómo puede

${ }^{34}$ Ibid., pp. 689-696.

35 Artículo 17. Proyecto de ley que "Regula los derechos y deberes que tienen las personas en relación con acciones vinculadas a su atención de Salud".

${ }^{36}$ Romeo, ob. cit., pp. 424-5. 
obligarse a una persona a recibir quimioterapia que no tendrá ningún éxito, y que sólo la hará sentirse degradada? ¿Cómo puede ser correcto mantener el sufrimiento de la familia, respecto de un ser querido que se sabe morirá? En este sentido, el proyecto de ley establece la posibilidad de no prolongar innecesariamente la agonía de un paciente terminal y dejar que su enfermedad siga su curso normal, hasta que se produzca su muerte, si así lo decide él”. 37

Con todo, considerar como diferentes el rechazo y la suspensión de un tratamiento es, para autores como R. G. Frey, un error pues en cada una de estas situaciones, argumenta, el médico ayuda a producir y ocasionar la muerte del enfermo como resultado de una petición de un individuo autónomo, capacitado e informado que desea que lo ayuden. Por ello, no le parece creíble clasificar como eutanasia pasiva aquella por la cual es preciso realizar una acción de desconexión o suspensión. “¿Cuál es la diferencia moral entre que el médico administre una pastilla que producirá la muerte y que retire los tubos de alimentación, con lo que también se producirá la muerte? Ambas cosas suceden como resultado de una petición del enfermo para que lo ayude a morir; ambas consiguen su objetivo; y en ambas el paciente y el médico colaboran para producir esta muerte (...) parece casi increíble que el hecho de que un paciente terminal esté o no conectado a una máquina pueda transformar los casos de tal modo desde un punto de vista moral, cuando ambos muestran con claridad que el enfermo y el médico actúan juntos para provocar la muerte del primero a petición suya". 38

Pero por increíble que parezca a R. G. Frey, lo cierto en que la clasificación y conceptualización revisada más arriba es admitida ampliamente por los principales organismos internacionales -Asociación Médica Mundial, Organización Mundial de la Salud, Consejo de Europa-, las legislaciones comparadas y la literatura especializada, que suele considerar, dentro del "derecho al consentimiento informado", el derecho a dar el consentimiento competente, desengañado y voluntario a someterse al tratamiento, como también el derecho a retirarse de ese mismo tratamiento en todo o parte. ${ }^{39}$ Recordemos que este fue el caso de Piergiorgio Welby, quien siendo enfermo terminal, suspendió su tratamiento. De igual modo en Chile la comunidad científica considera legítima la interrupción ("withdrawing") de los métodos extraordinarios de tratamiento cuando a) las técnicas empleadas imponen al paciente sufrimientos y molestias no tolerables para él; b) los resultados observados defraudan las esperanzas puestas en el procedimiento empleado; c) los médicos competentes juzgan que el uso del procedimiento es desproporcionado a la condición del paciente o a los resultados previsibles; d) el costo económico o emocional del tratamiento es claramente desmesurado para el paciente o la familia,

37 Mensaje del proyecto de ley que "Regula los derechos y deberes que tienen las personas en relación con acciones vinculadas a su atención de Salud".

38 Frey, ob. cit., pp. 58 y 59.

${ }^{39}$ Engelhardt, H. T., Los fundamentos de la bioética. Paidós. Barcelona, 1995. p. 327. 
y e) el justo deseo o voluntad del paciente y de sus familiares es que el procedimiento sea interrumpido. ${ }^{40}$

Pero vale la pena volver a la objeción de R.G. Frey. ¿Es distinto no iniciar un tratamiento que interrumpirlo? Se tiende a pensar que la omisión es menos causa de lo que por ella acontece o que es moralmente peor iniciar la cadena causal que tiene como resultado un daño (actuar) que no interrumpirla si ya ha empezado (omitir). Pablo de Lora se pregunta qué calificación ha de merecer la conducta de quien, convencido de la inutilidad de mantener los medios mecánicos de conservación de la vida, los desconecta. ¿Qué tipo de conducta es la de dejar de proporcionar un masaje cardiaco o interrumpir las sesiones de quimioterapia? Para De Lora se trata de casos en que se está ante un "dejar de hacer" y, por tanto, "eutanasia por omisión”. ${ }^{41}$ Ahora, parece inevitable, casi intuitivo, el reconocer que la desconexión de un respirador artificial-como en el caso de Piergiorgio Welby-implica, ya no una omisión, sino que una acción, cual es, la "acción de desconexión”. Parece inútil intentar distinguir actos y omisiones que en esencia parecen ser la misma cosa. Lo relevante son las consecuencias morales que derivemos de ello pues, se trate o no de una "acción” por parte del médico, resulta moralmente reprochable el obligar a una persona a mantener un tratamiento médico que le significa un gran sufrimiento, incluso aunque no se trate -como era el caso Inmaculada Echeverría- de un enfermo terminal. Esto es lo que implica reconocer a los pacientes el derecho al consentimiento informado.

¿Qué ocurre, ahora, con los casos en los que se aplican medicamentos a un paciente terminal con el fin de aliviar sus sufrimientos, aun cuando ello pueda acarrearle la muerte? Como vimos, para De Lora sería un tipo de eutanasia indirecta activa pues, aunque el móvil de la acción sea paliar el sufrimiento padecido -sabiendo que con gran probabilidad se acelerará la muerte- por definición es una "acción” pues no se puede, sin hacer nada, disminuir el dolor a alguien. El cuidado paliativo es una práctica asumida en todo el mundo civilizado pues se trataría de casos en los que no existe intención de matar, aunque sea ese el resultado de la acción. En la conciencia de la gente el propósito principal es mitigar el sufrimiento, no aumentarlo. ${ }^{42}$ Esto se relaciona con la llamada "doctrina del doble efecto" utilizada por Tomás de Aquino para justificar la muerte originada en la legítima defensa: "Nada impide que de un solo acto haya dos efectos, de los cuales uno sólo es intencionado y el otro no. Pero los actos morales reciben su especie de lo que está en la intención y no, por el contrario, de lo que es ajeno a ella (...) de la acción de defenderse puede entrañar un doble efecto: el uno es la conservación de la propia vida;

40 "El justo deseo del paciente, cuando le es posible manifestarlo, implica considerar varios elementos: la preeminencia de la voluntad del paciente por sobre la de los familiares; que el paciente no busque la muerte sino que desea evitar tratamientos que sólo lograrían una prolongación inútil y penosa de su vida; la aceptación de la muerte como un hecho inevitable de la vida humana. En lo que se refiere a los familiares, el médico debe abstenerse de acceder a sus solicitudes si percibe evidencias claras de que su actuación está en contradicción con el mejor interés del paciente”. Grupo de Estudio de Ética Clínica. op. cit., pp. 689-696.

${ }^{41}$ De Lora, P., ob. cit., pp. 79-80.

${ }^{42}$ De Lora, P., ob. cit., p. 83. 
el otro, la muerte del agresor (...) solamente es querido el uno; el otro, no (...) Tal acto, en lo que se refiere a la conservación de la propia vida, nada tiene de ilícito, puesto que es natural a todo ser conservar su existencia todo cuanto pueda". ${ }^{43}$

En el mismo sentido se ha manifestado el Grupo de Estudio de Ética Clínica de la Universidad Católica de Chile, al sostener que "Si del uso racional y técnicamente apropiado de analgésicos, indicados con la finalidad de aliviar el dolor y no con el propósito velado o declarado de acelerar la muerte del paciente, se sigue secundariamente el acortamiento de su vida, ello es lícito bajo el llamado principio del doble efecto o del voluntario indirecto. De acuerdo a este principio, una acción u omisión médica puede tener un doble efecto: uno positivo o bueno, que es el directamente querido, y otro negativo o malo, que sólo es tolerado al ser inevitable, pero no directamente deseado. En este caso, la acción médica de doble efecto será éticamente permitida en la medida que se cumplan las siguientes condiciones: a) Que la acción buscada sea buena en sí misma o, al menos, moralmente indiferente; b) Que quien actúa busque directamente el efecto bueno, siendo el eventual mal resultante secundario o colateral a la acción principal y no buscado ni deseado, y c) Que el efecto bueno buscado tenga tal importancia para la persona que lo recibe que resulta razonable o prudente la aceptación indirecta del mal secundario no evitable. ${ }^{44}$

Por cierto que no son pocas las críticas que pueden levantarse contra este tipo de razonamiento. El tema central es ¿puede un médico legítimamente, a petición de un paciente enfermo en fase terminal, administrarle morfina en cantidades tales que puedan conducir a la muerte a través de una depresión respiratoria? Como se revisó, la mayor parte de la comunidad científica argumenta que es legítimo siempre que la intención del médico sea aliviar el dolor y sufrimiento del enfermo, y no acelerar su muerte, aún cuando el médico sepa a ciencia cierta que el paciente morirá. Pero para autores como R.G. Frey, estas distinciones no pueden justificarse. "Algunas personas intentan cimentar una firme diferencia moral entre dos médicos y sus actos según la diferencia en su intención. Uno de ellos pretende matar al enfermo, el otro pretende aliviar su sufrimiento, consciente de la muerte del paciente que puede producirse o acelerarse (...) en el mencionado caso de la morfina. ¿De qué manera el médico podría ser causa parcial de esa muerte? Si administra una dosis alta de morfina que produce una depresión respiratoria y el enfermo muere, sería falso atribuir su muerte a un fallo respiratorio sin indicar lo que lo provocó, como lo sería achacar la muerte a un individuo al que le pasó un tren por encima sin indicar que alguien lo empujó a la vía”. ${ }^{45}$ Se trataría, nuevamente, de una distinción artificial que sólo pretende calificar de manera diferente hechos prácticamente idénticos.

En el mismo sentido se manifestaron Dworkin, Nagel, Nozick, Scanlon y Jarvis Thomson, a propósito del polémico caso de Nancy Cruzan. En su concepto, las clásicas

43 Tomás de Aquino, Suma Teológica. Espasa-Calpe, Buenos Aires, 1942. Parte II-II, cuestión 64, artículo 7 .

${ }^{44}$ Grupo de Estudio de Ética Clínica, op. cit., pp. 689-696.

45 Frey, R. G., ob. cit., pp. 46 y ss. 
distinciones entre actos y omisiones que pretenden diferenciar el suicidio asistido del derecho a interrumpir un tratamiento médico, no pueden justificarse moralmente. $\mathrm{Ni}$ desde el punto de vista del paciente existiría una diferencia moral pertinente entre los dos "actos" que acarrean finalmente la muerte, ni existe tampoco desde el punto de vista del médico. ${ }^{46}$ El desafío, entonces, es ser capaces de llamar las cosas por su nombre y, aún así, reconocer a las personas el derecho a decidir por sí mismas cuando un determinado tratamiento médico ha dejado de cumplir el objetivo para el cual habría sido previsto: mejorar la salud, aliviar el sufrimiento, salvar una vida que desea ser salvada. En caso contrario, cuando la medicina pasa a ser enemiga del paciente, el Estado debe ser capaz de reconocer los límites que separan el encarnizamiento terapéutico del legítimo cuidado sanitario. De lo contrario los pacientes dejan de ser sujetos de derecho para transformarse en objetos víctimas de la técnica médica.

\section{ii) ¿Quiénes son los titulares del derecho al consentimiento informado? Un nuevo derecho contra una vieja jurisprudencia}

Que los pacientes tienen, dentro del núcleo duro de lo que se entiende por libertad personal, derecho a decidir de forma general sobre su propio tratamiento -lo que implica necesariamente el derecho a otorgar o negar su consentimiento, después de haber recibido la información necesaria- implica que se trata de un derecho del que todos somos legítimos titulares, no sólo aquellos calificados como "enfermos terminales". Así, luego que el artículo 14 del proyecto reconoce el derecho en forma amplia ${ }^{47}$ el artículo 15 establecer taxativamente los únicos casos en los que no se requerirá la manifestación de voluntad: "No obstante lo establecido en el artículo anterior, no se requerirá la manifestación de voluntad en las siguientes situaciones: a) En caso que la falta de aplicación de los procedimientos, tratamientos o intervenciones señalados en el artículo anterior, supongan un riesgo para la salud pública, de conformidad a lo dispuesto en la ley, debiendo dejarse constancia al respecto en la ficha clínica de la persona; b) En aquellos casos en los que la condición de salud o cuadro clínico de la persona implique riesgo vital y/o secuela funcional grave de no mediar atención médica inmediata e impostergable, ésta no se encuentre en condiciones de expresar su voluntad, y no sea posible obtener el consentimiento de la persona que subrogue su decisión de acuerdo a lo establecido en el artículo siguiente; c) Cuando la persona se encuentra en incapacidad de manifestar su voluntad y no es posible obtenerla de su representante legal, por no existir o por no ser

46 “Assisted Suicide: The Philosophers' Brief” en The New York Review of Books, Vol. 44, No 5. 1997, p. 12 .

47 Artículo 14: "Toda persona tiene derecho a otorgar o denegar su voluntad para someterse a cualquier procedimiento o tratamiento vinculado a su atención de salud. El ejercicio de este derecho es libre, voluntario, expreso e informado (...)". Proyecto de Ley que "Regula los derechos y deberes que tienen las personas en relación con acciones vinculadas a su atención de Salud". 
habido. En estos casos se deberán tomar las medidas apropiadas sobre la base de lo que se conoce y de lo que es posible presumir acerca de la voluntad de la persona". ${ }^{48}$

En la misma línea, en el mensaje del proyecto se subraya que "El ejercicio de la autonomía de las personas respecto de su salud apunta a que éstas tienen el derecho a otorgar o denegar su voluntad para someterse a cualquier procedimiento. Este derecho, en principio, no tiene más limitaciones que excepciones basadas en razones de salud pública; también, en que los procedimientos médicos obedezcan a situaciones de emergencia, que no hagan posible recabar la voluntad de la persona (...)". ${ }^{49}$ Resulta comprensible el distinto tono que poseen los artículos 17 y 15 del proyecto en estudio pues, como se vio, el primero -que se ocupa del caso de los enfermos terminales- se refiere a aquella situación en que es necesaria la participación de un tercero, el médico, quien deberá interrumpir o suspender cierto tratamiento médico. En cambio en el segundo caso, contemplado en el artículo 15, no se requiere ningún acto de un tercero pues no se podría iniciar tratamiento alguno mientras no se recabe la voluntad del paciente -aun cuando su vida corra peligro. Luego, sólo el primero nos coloca en el caso de la mal llamada eutanasia "pasiva" o indirecta.

Aún así la disposición citada, de aprobarse en el Parlamento, debiera significar un rotundo cambio en nuestra jurisprudencia sobre derecho a la vida pues se deja claro que no es posible, por mor del respeto a la autonomía de las personas, obligar a nadie a someterse a tratamiento médico alguno, aun cuando sea para "protegerle de sí mismo" y salvarle la vida. Como se sabe, la jurisprudencia que nuestros tribunales de justicia mantienen sobre la protección del derecho a la vida garantizado en el artículo 19 $\mathrm{N}^{\circ} 1$ de la Constitución desconoce rotundamente un derecho como el "derecho al consentimiento informado", pues los tribunales de justicia han estimado, casi sin excepción, que la protección del derecho a la vida exigiría incluso obligar a determinadas personas a someterse a procedimientos médicos contra su voluntad. Este ha sido el caso de los Testigos de Jehová y de las personas que realizan huelgas de hambre. ${ }^{50}$

A modo de ejemplo se puede citar el caso paradigmático iniciado por el director del Hospital de Copiapó quien en 1992 habría recurrido de protección con el objeto de que se realizara, contra la voluntad del paciente Luis Muñoz Bravo - de 20 años y perteneciente a la religión de la Congregación Testigos de Jehová-, las transfusiones de sangre necesarias para salvarle la vida. La Corte Suprema, en su sentencia de confirmación, resolvió que "En consideración a la entidad del bien jurídico cuya protección se solicita y la necesidad urgente que existiría de cautelarlo, se ordena oficiar al señor Director del Hospital para que éste o quien lo subrogue disponga se aplique aun en contra de la

${ }^{48}$ Artículo 15. Proyecto de Ley que "Regula los derechos y deberes que tienen las personas en relación con acciones vinculadas a su atención de Salud".

49 Mensaje del Proyecto de Ley que "Regula los derechos y deberes que tienen las personas en relación con acciones vinculadas a su atención de Salud".

${ }^{50}$ Véase Zúñiga, A. "El interés público del derecho a la vida”, en González, Felipe (ed.), Litigio y Políticas Públicas en Derechos Humanos, Cuadernos de Análisis Jurídico, Serie de Publicaciones Especiales No 14, Universidad Diego Portales, Santiago, 2002, pp. 95-138. 
voluntad de don Luis Muñoz Bravo o de sus familiares, la terapia que sea necesaria para el tratamiento de la enfermedad que éste padece, incluida la transfusión sanguínea, pudiendo para ello recabar el auxilio de la fuerza pública en caso de oposición”, agregando que entre "el cotejo de los intereses en conflicto, el derecho a la vida y la libertad de conciencia, debe prevalecer el primero de los nombrados (...) Que, por otra parte, el juramento y rol profesional del médico lo obligan a preservar la vida". 51

En otro caso similar, el Director Ejecutivo Interino de la Fundación de Salud el Teniente dedujo un recurso de protección a favor de don Jorge Omar Reyes Ibarra, de 20 años, quien se encontraba internado en el Hospital de la fundación en estado de gravedad debido a que se habían negado terminantemente, él y su madre, a que se le efectuaran transfusiones de sangre por ser Testigos de Jehová. El fallo de la Corte de Apelaciones de Rancagua, que fue confirmado por la Corte Suprema el 22 de agosto de 1995, acogió el recurso al considerar que "Constituye una obligación de los Médicos tratantes de la persona en cuyo favor se ha incurrido, el procurar por todos los medios y técnicas que integran la lex artis médica el mantener la vida de sus pacientes (...) en razón de que debe primar la preservación de la salud y la vida de las personas sobre cualquiera otra consideración aunque sea de índole religiosa que ponga en riesgo innecesariamente la vida del enfermo" ${ }^{52}$ Similar razonamiento ha tenido la Corte Suprema en los casos de Matías Silva ${ }^{53}$ y de Patricia Castillo, ${ }^{54}$ acogiéndose en todos ellos los recursos de protección presentados.

La justificación moral de la práctica del consentimiento libre e informado radica en que respeta los diferentes criterios que existen sobre la dignidad individual, los valores asociados a la libertad de los individuos y el reconocimiento de que "cada cual es, por regla general, el mejor juez de lo que más le conviene e, incluso, si no lo fuere, reconoce que, por lo común, es preferible la satisfacción de elegir libremente, a elegir correctamente por imposición de otros". ${ }^{55}$ Uno de los más antiguos supuestos en los que se apoyan los derechos humanos se refiere a la protección del individuo en su derecho a que nadie toque su cuerpo sin su autorización. Así, es numerosa la jurisprudencia estadounidense que defiende este principio desde principios del siglo pasado. En el famoso caso Olmstead contra los Estados Unidos, de 1927, el juez Brandeis defendió que "Los padres de nuestra Constitución (...) intentaban proteger a los individuos americanos en sus creencias, sus pensamientos, sus emociones y sus sensaciones, confiriéndoles frente al gobierno el derecho a que se les deje en paz, el más amplio de los derechos y el que más valoran los hombres civilizados". 56

\footnotetext{
${ }^{51}$ Revista Fallos del Mes, No 402, Santiago, mayo 1992, pp. 227-231.

52 Revista Gaceta Jurídica, N 50, pág. 76, Santiago, 1995.

53 Revista Gaceta Jurídica, No 232, Santiago, octubre 1999, pp. 107-108.

54 Sentencia Corte Apelaciones de Santiago de $1^{\circ}$ de diciembre de 1995, confirmada por la Corte Suprema el 18 de enero de 1996.

55 Engelhardt, H. T., op. cit., p. 329.

${ }^{56}$ Olmstead v. Estados Unidos, 227 U.S. 438, 478 (1928). Voto disidente del juez Brandeis.
} 
De igual forma, el presidente del Tribunal Supremo de los Estados Unidos Warren Burger argumentó - a propósito del caso de una mujer que se negaba por motivos religiosos a una transfusión de sangre que le salvaría la vida- que "Nada sugiere en su pronunciamiento que el juez Brandeis pensase que un individuo posee estos derechos únicamente respecto a creencias sensatas, pensamientos válidos, emociones razonables o sensaciones bien fundamentadas. Me inclino a pensar que pretendía incluir muchas ideas insensatas, irrazonables y hasta absurdas, como la de rechazar tratamiento médico hasta en supuestos de gran riesgo". 57 En la misma línea, años después (1960) el tribunal del caso Natanson contra Kline argumenta que "Las leyes angloamericanas parten de la premisa de la autodeterminación total, de la cual se sigue que todo hombre es dueño de su propio cuerpo y puede, si está en su sano juicio, prohibir la práctica de toda cirugía tendiente a salvar su vida o de cualquier otro tratamiento médico". 58 Parece claro que por el hecho de que personas religiosas - como es el caso de los Testigos de Jehová- se comprometan en creencias que trascienden la razón o que incluso entren en conflicto con ella, no se puede invalidar su capacidad ni su derecho a que se respeten sus elecciones. "El que algunas elecciones individuales sean molestas, extrañas y trágicas para otros, no significa en sí mismo que se puede usar la fuerza para obstaculizarlas". 59

El proyecto de ley sobre Derechos del Paciente reconoce el derecho básico de toda persona a no ser obligado a someterse a determinados tratamientos, aun cuando de ello derive su muerte. El principio de autonomía prescribe que sólo con nuestro consentimiento se nos puede intervenir quirúrgicamente, suministrar un medicamento, administrar una terapia, conectar a un respirador artificial o introducir una sonda gástrica. ${ }^{60}$ Después de todo, esas son las exigencias de la libertad y del reconocimiento de la dignidad de todo ser humano.

\section{CONCLUSIÓN}

Uno de los más antiguos supuestos de las leyes inglesas se refiere a la protección del individuo en su derecho a que nadie toque su cuerpo sin su autorización. ${ }^{61}$ Los nuevos modos de morir asociados a los avances de la técnica médica nos obligan a reconocer con claridad aquellos derechos que, como el "consentimiento informado", se justifican por respeto a la libertad de individuo y exigen, antes de someter a alguien a algún tratamiento, obtener su permiso. El derecho al consentimiento libre e informado, en su acepción más fundamental, incluye el derecho a dar el consentimiento competente,

\footnotetext{
${ }^{57}$ Citado por Engelhardt, H. T., op. cit., p. 329.

58 Natanson v. Kline, 186 Kan. 393, 404, 350 P.2d 1093, 1104 (1960).

59 Engelhardt, op. cit., pp. 330.

${ }^{60}$ Corte Suprema de los Estados Unidos de Norteamérica. Caso Cruzan v. Missouri. 497 US 261,
} $269-270$.

${ }^{61}$ Engelhardt, ob. cit., p. 327. 
desengañado y voluntario a someterse al tratamiento como también el derecho a retirarse del mismo en todo o parte.

El proyecto de ley sobre derechos del paciente pendiente de discusión en el Congreso busca dicho reconocimiento haciéndose cargo adecuadamente, a nuestro entender, de los conflictos morales que la suspensión o interrupción de un tratamiento médico significa en el contexto del debate sobre la eutanasia. De igual forma, permite delimitar las esferas posibles y legítimas de acción del Estado en aquellos casos en los que los pacientes, por razones de conciencia, deciden no recibir un tratamiento médico necesario para salvar sus vidas. Ello, como se ha señalado, exigirá a los tribunales de justicia el reconocimiento del derecho básico a la autonomía y libertad que cada cual debe tener sobre el propio cuerpo.

\section{BIBLIOGRAFÍA}

Asociación Médica Mundial (AMM), Declaración de Lisboa sobre los Derechos del Paciente. 1981

De Lora, Pablo, Entre el vivir y el morir. Ensayos de Bioética y Derecho, Fontamara, México, 2003.

Engelhardt, H. Tristram, Los fundamentos de la bioética. Paidós. Barcelona, 1995.

Frey, R.G. "Distintos tipos de muerte", en La Eutanasia y el Auxilio Médico al Suicidio, Gerald Dworkin; R. G. Frey; Sissela Bok. Cambridge University Press, Madrid, 2000.

GRUPO DE ESTUdio DE ÉTICA CLÍNICA, Sobre las acciones médicas proporcionadas y el uso de métodos extraordinarios de tratamiento. Revista Médica de Chile, jun. 2003, Vol. 131, Nº 6, p. 689696.

Méndez, Víctor, "Eutanasias, derechos, razones”, Trotta, Madrid, 2001.

Organización Mundial de la Salud, Informe sobre la Salud en el Mundo 2000, Mejorar el desempeño de los sistemas de salud. Washington, 2001.

Organización Mundial de la Salud, Declaración para la promoción de los Derechos de los Pacientes en Europa. Amsterdam, 1994.

Romeo Casabona, Carlos María, "El derecho y la bioética ante los límites de la vida humana", Editorial Centro de Estudios Ramón Areces, Madrid, 1994.

Dworkin, Ronald, El Dominio de la Vida, Una discusión acerca del aborto, la eutanasia y la libertad individual, Ariel, Barcelona, 1994.

Dworkin, Nagel, Nozick, Rawls, Scanlon, Jarvis Thomson, "Assisted Suicide: The Philosophers' Brief" en The New York Review of Books, Vol. 44, N 5, 1997.

Singer, Peter, Ética práctica, Cambridge University Press, Gran Bretaña, 1995.

TABEADA, P., El principio de proporcionalidad terapéutica en las decisiones de limitar tratamientos. Boletín de la Escuela de Medicina de la Universidad Católica, 1998. 27:17-23.

Tomás de Aquino, Suma Teológica. Espasa-Calpe, Buenos Aires, 1942.

ZÚÑiga Fajuri, Alejandra, "El interés público del derecho a la vida" en González, Felipe (ed.) Litigio y Políticas Públicas en Derechos Humanos, Cuadernos de Análisis Jurídico, Publicaciones Especiales No 14, Universidad Diego Portales, Santiago, 2002. 